\title{
Erratum to: VOSalophen: a vanadium complex with a stilbene derivative-induction of apoptosis, autophagy, and efficiency in experimental cutaneous leishmaniasis
}

\author{
Patrícia de A. Machado ${ }^{1}$ Jessica O. F. Moraes ${ }^{2} \cdot$ Gustavo S. G. Carvalho $^{3}$. \\ Wallace P. Lima ${ }^{4}$ Gilson C. Macedo ${ }^{1}$ Elizandra A. Britta ${ }^{5}$ Celso V. Nakamura ${ }^{5}$. \\ Adilson D. da Silva ${ }^{3} \cdot$ Alexandre Cuin $^{2} \cdot$ Elaine S. Coimbra $^{1}$
}

Published online: 18 July 2017

(C) SBIC 2017

\section{Erratum to: J Biol Inorg Chem}

DOI 10.1007/s00775-017-1471-2

In the original publication, the second author's name was incorrectly published as Jessica O. F. Morais. The correct name should read as 'Jessica O. F. Moraes'.

The online version of the original article can be found under doi:10.1007/s00775-017-1471-2.

Elaine S. Coimbra

elaine.coimbra@uff.edu.br

1 Departamento de Parasitologia, Microbiologia e Imunologia, Instituto de Ciências Biológicas, Universidade Federal de Juiz de Fora, Juiz de Fora, MG 36036-900, Brazil

2 LQBin, Laboratório de Química BioInorgânica, Departamento de Química, Instituto de Ciências Exatas, Universidade Federal de Juiz de Fora, Juiz de Fora, MG 36036-900, Brazil

3 Departamento de Química, Instituto de Ciências Exatas, Universidade Federal de Juiz de Fora, Juiz de Fora, MG 36036-900, Brazil

4 Laboratório de Imunofarmacologia, Instituto de Biofísica Carlos Chagas Filho, Universidade Federal do Rio de Janeiro, Rio de Janeiro 21941-902, Brazil

5 Programa de Pós-Graduação em Ciências Biológicas, Universidade Estadual de Maringá, Maringá, Paraná 87020-900, Brazil 